\title{
Ascorbic acid supplementation does not attenuate post-exercise muscle soreness following muscle-damaging exercise but may delay the recovery process
}

\author{
Graeme L. Close ${ }^{1}$, Tony Ashton ${ }^{2}$, Tim Cable $^{1}$, Dominic Doran ${ }^{1}$, Chris Holloway $^{1}$, Frank McArdle ${ }^{2}$ \\ and Don P. M. MacLaren ${ }^{1}$ \\ ${ }^{1}$ Research Institute for Sport and Exercise Sciences, Liverpool John Moores University, Henry Cotton Campus, 15-21 \\ Webster Street, Liverpool L3 2ET, UK \\ ${ }^{2}$ School of Clinical Sciences, Division of Metabolic and Cellular Medicine, University of Liverpool, Liverpool L69 $3 G A$, UK
}

(Received 7 October 2005 - Revised 15 December 2005 - Accepted 19 December 2005)

\begin{abstract}
Exercise involving lengthening muscle actions, such as downhill running, results in delayed onset muscle soreness (DOMS), which may be attributable to reactive oxygen species (ROS). Although exercise causes oxidative stress, any link between ROS and DOMS remains speculative. There is emerging evidence to suggest that ROS play an important physiological role, assisting in the recovery process and protecting the cell from future damage; however, this has not been fully established. Despite this uncertainty as to the precise role of ROS, attempts to prevent post-exercise ROS production through antioxidant intervention are still common. The study investigated the effects of ascorbic acid supplementation on ROS production and DOMS following downhill running. Subjects were assigned to two groups. The ascorbic acid group (group AA) received $1 \mathrm{~g}$ ascorbic acid $2 \mathrm{~h}$ pre-, and for $14 \mathrm{~d}$ post-downhill running, whilst the placebo group (Pl group) received a placebo. Blood samples were drawn pre-supplement, pre- and post-exercise, and then 1, 2, 3, 4, 7 and 14d post-exercise for analysis of ascorbate, malonaldehyde and total glutathione. DOMS was assessed using a visual analogue scale and pressure algometry. Muscle function was assessed using isokinetic dynamometry. Plasma ascorbate was elevated throughout in group AA compared with the Pl group. Downhill running resulted in DOMS in both groups. Muscle function was impaired post-exercise in both groups, although a delayed recovery was noted in group AA. Malonaldehyde increased $4 \mathrm{~d}$ post-exercise in the Pl group only. Ascorbic acid supplementation attenuates ROS production following downhill running, without affecting DOMS. Furthermore, ascorbic acid supplementation may inhibit the recovery of muscle function.
\end{abstract}

Reactive oxygen species: Exercise: Eccentric muscle torque: Antioxidants: Muscle damage

Unaccustomed or excessive exercise often results in the sensation of muscular discomfort and pain that is characterised by its delay in onset and has been termed 'delayed onset muscle soreness' (DOMS) (Newham et al. 1983). DOMS is often first noticed $24 \mathrm{~h}$ post-exercise and presents as a dull ache similar to that of a bruise. Despite extensive research into DOMS, the underlying causes and methods of prevention remain unresolved (Close et al. 2005).

We have previously demonstrated that $30 \mathrm{~min}$ of downhill running at a sub-maximal intensity results in muscle damage and a significant increase in reactive oxygen species (ROS) production and subsequent lipid peroxidation in the days following the exercise (Close et al. 2004). This increase in lipid peroxidation was speculated to be due to phagocytederived superoxide $\left(\mathrm{O}_{2}{ }^{--}\right)$production resulting in the subsequent formation of the more potent hydroxyl radical $(\mathrm{OH})$. However, it is still unclear if this production of ROS in the days following lengthening muscle contractions is a pathological process that amplifies the sensations of DOMS, or is an essential physiological process assisting in the recovery from the initial trauma. The reason for this is that merely the presence of ROS cannot determine if their production is involved in the observed pathology. The only way that ROS can be confirmed to be involved in the pathology is through specific intervention (Jackson, 1999). Despite this uncertainty as to the exact role of ROS following contraction-induced damage, it is common practice for athletes to use antioxidant therapy to prevent post-exercise ROS production and, furthermore, there is still extensive research investigating ways to prevent DOMS and muscle damage using antioxidant supplementation (Jakeman \& Maxwell, 1993; Goldfarb, 1999; Thompson et al. 2001a).

Ascorbic acid is a water-soluble, dietary antioxidant present in the cytosolic compartment of the cell and the extracellular fluid, and is known to be a powerful inhibitor of lipid peroxidation (Powers et al. 2004), in conjunction with $\alpha$-tocopherol (Evans, 2000). The spatial arrangement of ascorbic acid allows it to scavenge aqueous-phase $\alpha$-tocopherol radicals 
generated in the cell membrane during oxidative stress, through its ability to rapidly donate electrons to $\alpha$-tocopherol radicals. In response to physical trauma, ascorbic acid is able to provide protection against phagocyte-mediated cell injury by scavenging the specific phagocyte-derived ROS (Nieman et al. 2002). Ascorbic acid is able to scavenge phagocytederived ROS by rapid aqueous-phase electron transfer and thereby reducing the adhesion of phagocytes to the endothelium, attenuating their respiratory burst and preventing subsequent lipid peroxidation (Kearns et al. 2001).

The aim of the present study was to examine the effects of prolonged ascorbic acid supplementation on ROS production and investigate the effects of this on DOMS and muscle function following downhill running. It was hypothesised that supplementation with ascorbic acid would attenuate post-exercise increases in ROS production, reduce the magnitude of DOMS and affect the recovery of muscle function.

\section{Subjects and methods}

\section{Subjects}

Twenty physically active male subjects, naive to downhill running, volunteered for the study. All were non-smokers and free from any known illnesses as ascertained by questionnaire. Subjects taking any form of vitamin supplementation were excluded. All subjects were informed verbally and in writing about the nature of the study, including all potential risks. Written informed consent was obtained before participation and the ethics committee of Liverpool John Moores University (Liverpool, UK) granted ethical approval. The chosen subjects were instructed to avoid any form of therapeutic intervention throughout the trial including antioxidants, anti-inflammatory drugs, ice and massage therapy.

\section{Experimental protocol}

Each subject was initially assessed for aerobic fitness by determining their $\mathrm{V}_{\mathrm{O} 2 \max }$, as described previously (Close et al. 2004). Assessment of $\mathrm{V}_{\mathrm{O} 2 \max }$ was carried out 1 week before the downhill run. The downhill run lasted for $30 \mathrm{~min}$ at a running speed corresponding to $60 \% \mathrm{~V}_{\mathrm{O} 2 \max }$. All subjects were randomly allocated into two groups; placebo group (Pl group; $n$ 10), and ascorbic acid group (group AA; $n$ 10).

For each trial, subjects were required to visit the laboratories on seven occasions. Day 1 was to receive the supplement, have muscle function and pain measurements taken, provide pre- and post-supplement venous blood samples, and perform the downhill run and to give a post-exercise venous blood sample. The following $4 \mathrm{~d}$ were to have muscle function and pain assessed, and to give a resting venous blood sample. Subjects were required to visit the laboratory again on day 7 and day 14 to have muscle function and pain assessed, and to give a resting venous blood sample. Subjects were instructed to avoid any strenuous exercise for $3 \mathrm{~d}$ before the downhill run, and to continue to abstain from any strenuous exercise throughout the $14 \mathrm{~d}$ duration of the test. Subjects were also requested to avoid any form of therapeutic intervention to alleviate the muscle soreness including, drug, ice or massage therapy.
Day 1

On the day of the experimental run, subjects reported at the laboratory $2 \mathrm{~h}$ before their allocated run time. On arrival subjects were seated for $30 \mathrm{~min}$ and then a pre-supplement blood sample was taken. Subjects were then given either ascorbic acid (1 g) (Roche, Lewes, East Sussex, UK) or a visually identical lactose placebo (1 g) (Roche). Subjects were randomly assigned into the two groups and supplementation was administered double blind. Following the supplementation, subjects were given a $2 \mathrm{~h}$ break to allow time for the supplement to be absorbed (Thompson et al. 2001a).

Subjects returned to the laboratory $2 \mathrm{~h}$ post-supplementation to give a second venous blood sample and were then asked to rate their pre-exercise muscle soreness of the gastrocnemius, anterior tibialis, hamstrings, quadriceps, gluteals (both left and right sides) and lower back muscles using a visual analogue scale and pressure algometry. Subjects had been previously familiarised with both of the methods of pain assessment to eliminate any learning effects. Subjects then proceeded to warm up on a cycle ergometer for $5 \mathrm{~min}$ before completing a series of stretches of their own choice. The stretches were to prepare them for the assessment of muscle function on an isokinetic dynamometer, as described previously (Close et al. 2004). Briefly, the test involved concentric quadriceps muscle torque assessment at 1.06 and $5.20 \mathrm{rad} / \mathrm{s}$ as well as eccentric quadriceps assessment at $2.6 \mathrm{rad} / \mathrm{s}$ and all subjects had been fully familiarised with the strength assessments before the start of the experiments.

All blood samples were collected in pre-treated vacutainers following an overnight fast from a superficial vein in the antecubital fossa whilst the subjects were supine and had been resting for a minimum of $30 \mathrm{~min}$ (except for the post-exercise sample, which was taken immediately upon cessation of exercise). Samples were taken without stasis to prevent any potential increase in oxidative stress due to ischaemic-reperfusion injury. A total of four vacutainers were used at each sample point.

Tube $1(5 \mathrm{ml})$ contained the anticoagulant EDTA and was analysed for total blood glutathione, packed cell volume and $\mathrm{Hb}$. Of this $5 \mathrm{ml}$ whole blood, $100 \mu \mathrm{l}$ was immediately deproteinised with $500 \mu \mathrm{l}$ hydrochloric acid $(10 \mathrm{mM})$, before being vortex mixed for $30 \mathrm{~s}$ and $300 \mu \mathrm{l}$ cold $10 \%$ 5-sulfosalicylic acid added. The sample was vortex mixed for a further $30 \mathrm{~s}$ before being spun at $10000 \mathrm{rpm}$ for $5 \mathrm{~min}$ and the supernatant fraction removed and stored at $-70^{\circ} \mathrm{C}$. This was later analysed for total glutathione content (Anderson, 1985). The remaining EDTA-treated blood was analysed within $24 \mathrm{~h}$ for packed cell volume and $\mathrm{Hb}$ using an automated Coulter counter (Coulter MaxM; Beckman Coulter, Fullerton, CA, USA).

Tube $2(6 \mathrm{ml})$ contained serum separation gel and was used for the determination of malonaldehyde (MDA) using HPLC (Gina 50; Gynkotek, Macclesfield, Cheshire, UK) as described by Richard et al. (1992).

Tube $3(6 \mathrm{ml})$ contained lithium heparin and was used for the analysis of plasma ascorbate. The tube was immediately centrifuged and a sample of plasma $(0.6 \mathrm{ml})$ was immediately added to $0.6 \mathrm{ml} 10 \%$ metaphosphoric acid (Sigma Chemicals Co. Ltd, Poole, Dorset, UK), vortex mixed and then immediately stored at $-80^{\circ} \mathrm{C}$ for later analysis of 
ascorbic acid using HPLC (Gina 50; Gynotek) according to the method of Mohr \& Stocker (2002).

The downhill run took place on the same motorised treadmill used in the $\mathrm{V}_{\mathrm{O} 2 \max }$ test. The run lasted for $30 \mathrm{~min}$ at a decline of $-15 \%$ and at an $\mathrm{O}_{2}$ consumption corresponding to about $60 \% \mathrm{~V}_{\mathrm{O} 2 \mathrm{max}}$. Throughout the trial, expired gas volumes and concentrations were recorded every $10 \mathrm{~s}$ (Metamax; Cortex Biophysic GmbH, Leipzig, Germany). The treadmill speed was continually altered to ensure that the $\mathrm{O}_{2}$ consumption corresponding to about $60 \% \mathrm{~V}_{\mathrm{O} 2 \max }$ was maintained. Every $10 \mathrm{~min}$, ratings of perceived exertion were recorded (6-20 Borg Scale), as well as heart rate using a short-range radio telemeter (Sportstester PE3000; Polar Electro, Kempele, Finland). Thermal comfort was maintained throughout the run by means of two electronic fans (MS16; Amcon Controls Inc., San Antonio, TX, USA).

Immediately following the run, subjects assumed a supine position whilst a third blood sample was taken using the same technique as described previously. Subjects were then re-assessed regarding their perceptions of muscle soreness before completing the force assessment again. This completed day 1 of the trial. All subjects were then instructed to continue to take their allocated supplement for $14 \mathrm{~d}(1 \mathrm{~g} / \mathrm{d})$.

\section{Days 2, 3, 4, 5, 7 and 14}

Subjects visited the laboratory exactly $1,2,3$ and $4 \mathrm{~d}$ after their initial visit and again at day 7 and day 14 post-exercise following an overnight fast. Subjects were instructed to assume a supine position for $30 \mathrm{~min}$ before a resting blood sample was taken from a prominent vein. Following the blood tests, subjects were then assessed for DOMS. This was followed by a $5 \mathrm{~min}$ warm-up on a cycle ergometer before muscle function was re-assessed.

\section{Statistical analyses}

Statistical analyses were carried out using the Statistical Package for Social Sciences (version 11.01; SPSS, Woking, Surrey, UK). All data are presented as means with their standard errors. Data were analysed using Student's $t$ test. Where multiple comparisons where made, data were analysed using a mixed design ANOVA to prevent family-wise errors. When Mauchley's test of sphericity indicated a minimum level of violation (Greenhouse Geisser $\epsilon>0.75$ ) data were corrected using the Huynh-Feldt $\epsilon$; however, when sphericity was violated (Greenhouse Geisser $\epsilon<0.75$ ) data were corrected using the Greenhouse Geisser $\epsilon$ (Field, 1999). Where significant $F$ values were observed, Tukey's honestly significant difference post hoc analysis was performed to identify where the significant differences occurred. Statistical significance was set at the $\alpha$ level of 0.05 for all tests.

\section{Results}

\section{Subject information}

Before the experimental runs, subjects were initially assessed for $\mathrm{V}_{\mathrm{O} 2 \mathrm{max}}$ as well as having their height, weight, and percentage body fat calculated. There was no significant difference between the two groups for any of the characteristics (two sample $t$ test; $P>0.05$ ). A summary of the subject characteristics can be seen in Table 1. All subjects successfully completed the $30 \mathrm{~min}$ run at the desired work intensity. There were no significant differences $(P>0.05)$ in heart rates, $\mathrm{V}_{\mathrm{O} 2 \max }$, ratings of perceived exertion, RER, minute ventilation and run speed between the two groups.

\section{Plasma ascorbate concentration}

There was no significant difference in baseline plasma ascorbate concentration between the two groups $(P>0 \cdot 05)$. However, $2 \mathrm{~h}$ post-supplementation, plasma ascorbate had significantly increased in group AA compared with the Pl group $(P<0 \cdot 05)$. Furthermore, plasma ascorbate concentrations remained significantly elevated above basal values for the 2 -week period in group AA $(P<0 \cdot 05)$. There was a significant increase in plasma ascorbate concentration in the $\mathrm{Pl}$ group immediately post-exercise $(P<0 \cdot 05)$. Plasma ascorbate concentrations for group AA and the $\mathrm{Pl}$ group throughout the trial can be seen in Fig. 1.

\section{Delayed onset muscle soreness}

Ratings of DOMS are presented as mean soreness for the eleven assessed sites. The downhill runs were successful in eliciting significant muscle soreness in both group AA and the Pl group. Peak soreness occurred $24-48 \mathrm{~h}$ post-exercise in both groups and was still significantly elevated $96 \mathrm{~h}$ postexercise $(P<0.05)$. This was evident in the visual analogue scale (Fig. 2 (A)), and pressure algometry (Fig. 2 (B)). All perceptions of DOMS had returned to baseline by $14 \mathrm{~d}$ post-exercise and there were no significant differences at any of the time points between the two groups $(P>0.05)$.

\section{Reactive oxygen species}

There was no increase in MDA concentration in both groups immediately post-exercise $(P>0.05)$. There was a

Table 1. Characteristics of the two subject groups

(Mean values with their standard errors)

\begin{tabular}{|c|c|c|c|c|c|c|c|c|c|c|}
\hline & \multicolumn{2}{|c|}{ Age (years) } & \multicolumn{2}{|c|}{ Height (cm) } & \multicolumn{2}{|c|}{ Weight (kg) } & \multicolumn{2}{|c|}{$\mathrm{V}_{\mathrm{O} 2 \max }($ litres/min) } & \multicolumn{2}{|c|}{ Body fat (\%) } \\
\hline & Mean & SEM & Mean & SEM & Mean & SEM & Mean & SEM & Mean & SEN \\
\hline Placebo group & $22 \cdot 1$ & 0.4 & 180 & 1.6 & 78.4 & 4.6 & $4 \cdot 3$ & 0.2 & $14 \cdot 2$ & $1 \cdot 1$ \\
\hline Ascorbic acid group & $24 \cdot 2$ & 1.5 & 179 & 1.9 & $78 \cdot 3$ & 1.9 & $4 \cdot 4$ & 0.1 & $13 \cdot 6$ & 0.9 \\
\hline
\end{tabular}

For details of subjects and procedures, see p. 977. 


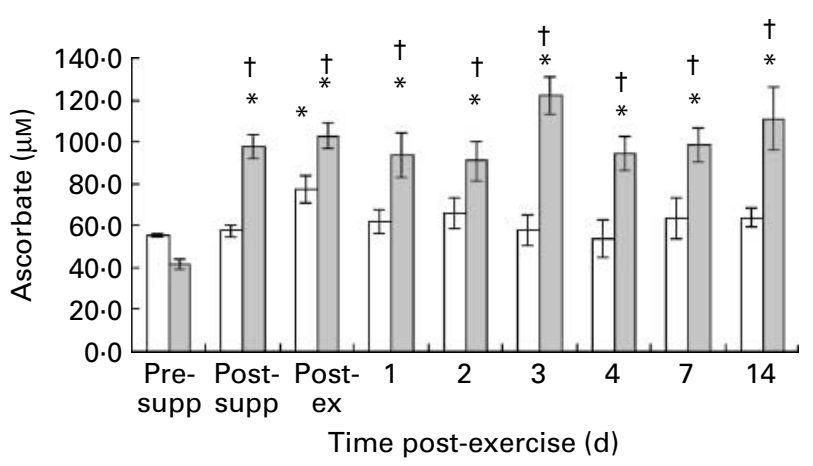

Fig. 1. Plasma ascorbate concentrations of the placebo group ( $\square$ ) and ascorbic acid group $(\square)$. Values are means, with their standard errors represented by vertical bars. Supp, supplement; ex, exercise. * Mean value was significantly different from that pre-supp $(P<0.05)$. † Mean value was significantly different from that of the placebo group $(P<0.05)$. For details of subjects and procedures, see p. 977.

significantly elevated serum MDA concentration in the $\mathrm{Pl}$ group compared with group AA at 72 and $96 \mathrm{~h}$ post-exercise $(P<0.05)$, increasing from 0.80 (SEM 0.04$) \mu \mathrm{M}$ pre-exercise to 1.09 (SEM 0.05) and 1.13 (SEM 0.07) $\mu \mathrm{M}$ at 72 and $96 \mathrm{~h}$ post-exercise respectively. This increase was transient and there was no significant difference from pre-exercise by day 7 post-exercise $(0.92$ (SEM 0.04$) \mu \mathrm{M})$. There was no significant change in total glutathione concentration across time or between the two groups $(P>0 \cdot 05)$.

\section{Muscle function}

Muscle function is expressed as percentage change in torque from pre-exercise scores and is summarised in Table 2.
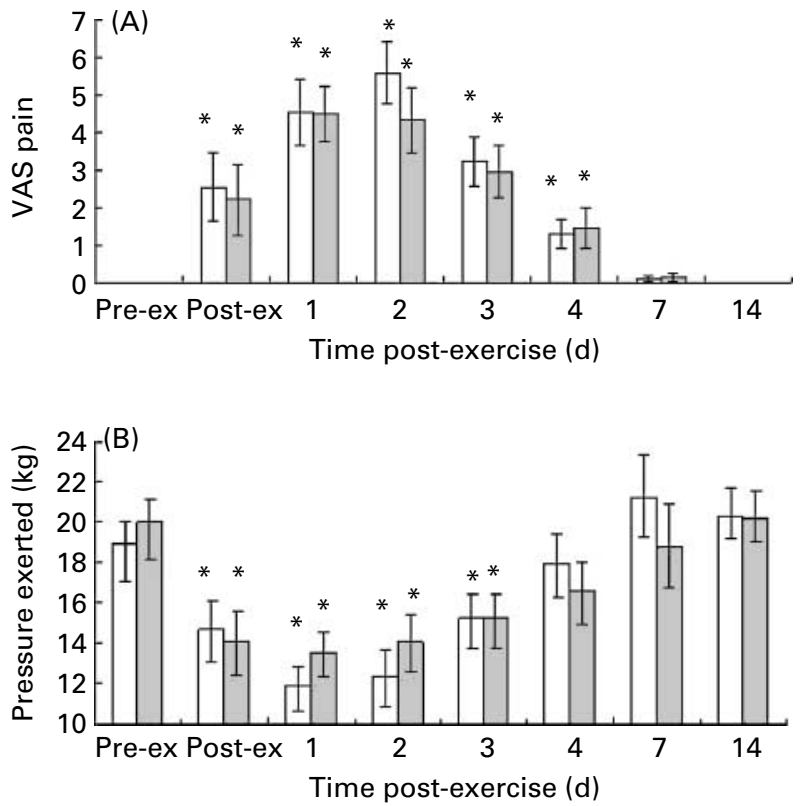

Fig. 2. Ratings of muscle soreness for the placebo group ( $\square$ ) and ascorbic acid group ( $\square$ ) assessed using a visual analogue scale (VAS) of $0-10(A)$ and using pressure algometry (B). Data are presented as the summed pain scores or the summed forces divided by the number of sites assessed. ex, exercise. Values are means, with their standard errors represented by vertical bars. "Mean value was significantly different from that pre-exercise $(P<0.05)$. For details of subjects and procedures, see p. 977.

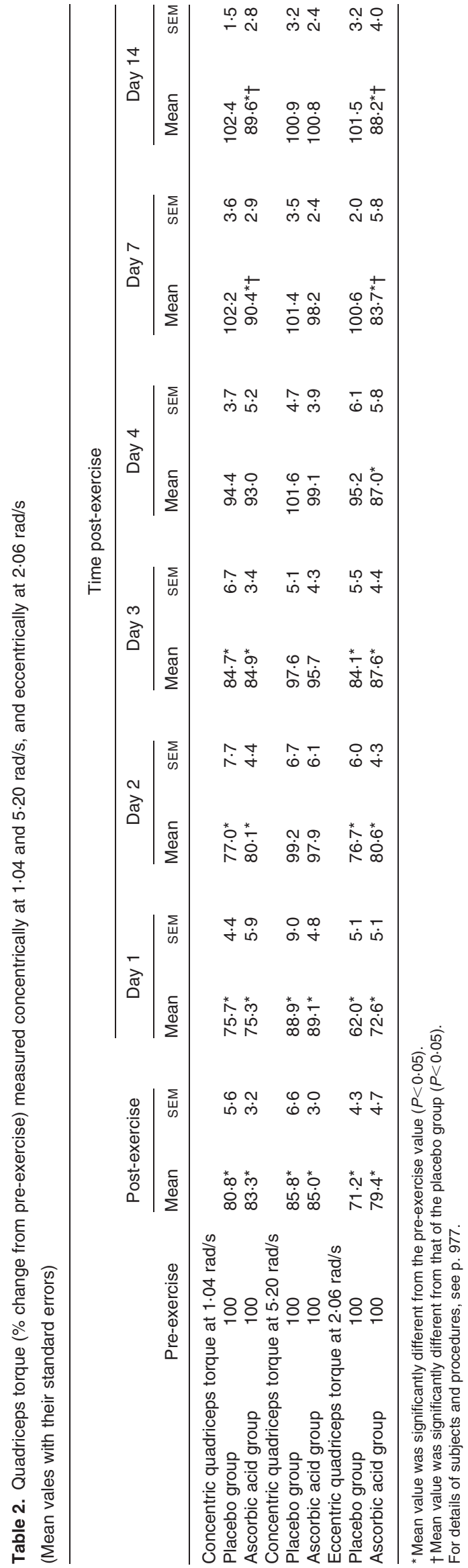


A significant loss of peak torque measured at $1.04 \mathrm{rad} / \mathrm{s}$ was observed post-exercise in both groups $(P<0 \cdot 05)$. In the $\mathrm{Pl}$ group muscle function returned to basal levels by day 7 $(P>0.05)$, although in group AA muscle function was still significantly impaired at both 7 and $14 \mathrm{~d}$ post-exercise $(P<0.05)$ and had not returned to basal levels by the end of the test period.

Muscle function was also impaired in both groups postexercise when measured at $5.20 \mathrm{rad} / \mathrm{s}$. There was, however, no significant difference between the two groups $(P>0.05)$, with muscle function returning to basal levels by $4 \mathrm{~d}$ postexercise in both groups.

Eccentric muscle torque at $2.06 \mathrm{rad} / \mathrm{s}$ was also assessed. There was a significant loss of muscle torque post-exercise in both groups $(P<0 \cdot 05)$. This was still apparent up to $4 \mathrm{~d}$ post-exercise in both groups. However, although muscle torque had returned to basal levels by day 4 in the Pl group $(P>0.05)$, muscle torque was still impaired at day 7 and day 14 post-exercise in group AA.

\section{Discussion}

The present study investigated the effects of ascorbic acid supplementation on ROS production, DOMS and muscle function following downhill running. The results clearly show that ascorbic acid supplementation increased plasma ascorbate concentrations, resulting in attenuated increases in serum MDA concentration compared with a placebo. MDA is a marker of ROS-mediated lipid peroxidation, therefore the present data suggest that ascorbate is able to scavenge the excess production of ROS before lipid peroxidation occurs. Although attenuation of ROS following ascorbic acid supplementation has been demonstrated immediately post-exercise following shuttle running (Thompson et al. 2001a), this is the first report of ascorbic acid attenuating ROS in the days following muscle-damaging exercise.

It is known that MDA correlates with a lipid radical (alkoxyl or alkyl radical) as detected by electron spin resonance spectroscopy (Davison, 2002), and furthermore this lipid radical is thought to represent ${ }^{\circ} \mathrm{OH}$ attack on lipid membranes (Pattwell et al. 2003). The increase in MDA in the present study is therefore likely to be related to phagocyticderived $\mathrm{O}_{2}{ }^{--}$production leading to ${ }^{\circ} \mathrm{OH}$ generation through Fenton chemistry. Ascorbate is able to specifically protect against phagocytic-derived $\mathrm{O}_{2}{ }^{--}$through its ability to rapidly donate an electron to $\mathrm{O}_{2}^{--}(\mathrm{Ji}, 1995)$. It would therefore appear that $1 \mathrm{~g}$ ascorbic acid given before and following downhill running successfully saturates phagocytes with ascorbate and attenuates the oxidative burst. Alternatively, $1 \mathrm{~g}$ supplements of ascorbic acid may cause an increase in plasma ascorbate concentrations that is sufficient to effectively deal with the increased ROS production. This ultimately prevents the peroxidation of lipid membranes and accounts for the decreased serum MDA production.

The present study sought to clarify the effect of ascorbic acid supplementation on DOMS. There was no significant difference in DOMS between the two groups despite ascorbic acid supplementation significantly increasing plasma ascorbate concentrations. Peak pain occurred $2 \mathrm{~d}$ post-exercise and all perceptions of DOMS had gone by day 7. It was also observed that although antioxidant therapy reduced ROS production, supplementation with ascorbic acid had no effect on DOMS, further suggesting dissociation between ROS and DOMS.

Muscle function was assessed by measuring changes in muscle torque on an isokinetic dynamometer. Concentric quadriceps torque at $1.04 \mathrm{rad} / \mathrm{s}$ fell significantly in both groups post-exercise. In the $\mathrm{Pl}$ group, muscle torque returned to pre-exercise levels at $96 \mathrm{~h}$ post-exercise; however, in group AA, muscle torque was still significantly less than pre-exercise at day 7 and day 14 post-exercise. A similar pattern was also seen when muscle torque was assessed eccentrically at 2.60 $\mathrm{rad} / \mathrm{s}$. These results suggest that ROS produced as a consequence of muscle-damaging exercise may play a vital role in muscle regeneration and that supplementation with ascorbic acid is detrimental to the recovery. These findings are supported by Thompson et al. (2001b), who also reported attenuated muscle torque in the leg flexors following ascorbic acid supplementation when assessed at $1.04 \mathrm{rad} / \mathrm{s}$.

One explanation for this prolonged loss of muscle function is that attenuating the oxidative burst may hinder phagocytic cells from killing and removing the damaged and necrotic tissue. It is known that phagocytosis occurs following muscle-damaging exercise, specifically involving the infiltration of neutrophils and macrophages into the damaged site. Once microbes, particulate material or cytokines perturb the membrane of the phagocyte, these cells then produce $\mathrm{O}_{2}{ }^{--}$, a process referred to as the oxidative burst (Leeuwenburgh $\&$ Heinecke, 2001). Further redox reactions can result in the formation of $\mathrm{HOCl}^{-}$and $\mathrm{OH}$. It is suspected that $\mathrm{HOCl}^{-}$ and $\mathrm{OH}$ are the important end points of this oxidative burst (Niess et al. 1999), although this is still not fully established. The present study demonstrated that $1 \mathrm{~g}$ ascorbic acid supplementation prevents post-exercise ROS production and this in turn may delay the recovery of muscle function.

It has been suggested that in high concentrations $(>500 \mathrm{mg})$ and in the presence of transition metals such as $\mathrm{Fe}^{3+}$ or $\mathrm{Cu}^{2+}$ ascorbic acid may act as a pro-oxidant as well as an antioxidant (Podmore et al. 1998). The reason for the pro-oxidant action of vitamin $\mathrm{C}$ is due to its ability to reduce ferric $\mathrm{Fe}$ $\left(\mathrm{Fe}^{3+}\right)$ to the ferrous state $\left(\mathrm{Fe}^{2+}\right)$ (Powers et al. 2004) and $\mathrm{Fe}^{2+}$ is known to be an important catalyst in the production of ROS, for example in the Fenton reaction (Cheeseman \& Slater, 1993). It could therefore be suggested that the delayed recovery observed in group AA could be due to the pro-oxidant action of ascorbic acid. However, in a recent review on the pro-oxidant potential of ascorbic acid, it was suggested that, overall, the literature does not support the suggestion that ascorbic acid is a pro-oxidant in vivo (Carr \& Frei, 1999), blaming oxidation artifacts for the reports that have shown a pro-oxidant affect of ascorbic acid. Furthermore, in the present study there was no evidence of increased oxidative stress in group AA compared with the Pl group and, consequently, it appears unlikely that the delayed recovery is due to a pro-oxidant action of ascorbic acid.

The findings of the present study have significant implications for both clinicians and athletes. Contrary to popular belief, ROS produced in the days following muscle-damaging exercise may not be responsible for the prolonged losses of muscle function and DOMS and, conversely, they may play a key role in mediating the recovery. Moreover, supplementation with ascorbic acid to prevent post-exercise ROS production does not attenuate DOMS or preserve muscle 
function, but may hinder the recovery process. Therefore, supplementation of ascorbic acid before and following muscle-damaging exercise is not only unnecessary, but may be detrimental to future performance.

\section{Acknowledgements}

The authors would like to thank Dr Anne McArdle, Professor Malcolm Jackson (University of Liverpool) and Michael Gleeson (Loughborough University) for useful scientific discussions, Mr Lee Siemesko (Liverpool John Moores University) for his assistance in the data collection and all subjects for their efforts.

\section{References}

Anderson M (1985) Determination of glutathione and glutathione disulphide. Methods Enzymol 113, 548-555.

Carr A \& Frei B (1999) Does vitamin C act as a pro-oxidant under physiological conditions? FASEB J 13, 1007-1024.

Cheeseman KH \& Slater TF (1993) An introduction to free radical biochemistry. Br Med Bull 49, 481-493.

Close GL, Ashton T, Cable T, Doran D \& MacLaren DP (2004) Eccentric exercise, isokinetic muscle torque and delayed onset muscle soreness: the role of reactive oxygen species. Eur J Appl Physiol 91, 615-621.

Close GL, Ashton T, McArdle A \& MacLaren DP (2005) The emerging role of free radicals in delayed onset muscle soreness and contractioninduced muscle injury. Comp Biochem Physiol 142A, 257-266.

Davison G (2002) Exercise and oxidative stress: implications in health and disease. PhD Thesis, University of Glamorgan, School of Applied Sciences, Pontypridd, Wales, UK.

Evans WJ (2000) Vitamin E, vitamin C, and exercise. Am J Clin Nutr 72, Suppl. 2, 647S-652S.

Field A (1999) Discovering Statistics using SPSS for Windows. London: Sage Publications.

Goldfarb AH (1999) Nutritional antioxidants as therapeutic and preventive modalities in exercise-induced muscle damage. Can J Appl Physiol 24, 249-266.

Jackson MJ (1999) An overview of methods for assessment of free radical activity in biology. Proc Nutr Soc 58, 1001-1006.

Jakeman P \& Maxwell S (1993) Effect of antioxidant vitamin supplementation on muscle function after eccentric exercise. Eur J Appl Physiol Occup Physiol 67, 426-430.
Ji LL (1995) Exercise and oxidative stress: role of the cellular antioxidant systems. Exerc Sport Sci Rev 23, 135-166.

Kearns SR, Moneley D, Murray P, Kelly C \& Daly AF (2001) Oral vitamin $\mathrm{C}$ attenuates acute ischaemia-reperfusion injury in skeletal muscle. J Bone Joint Surg Br 83, 1202-1206.

Leeuwenburgh C \& Heinecke JW (2001) Oxidative stress and antioxidants in exercise. Curr Med Chem 8, 829-838.

Mohr D \& Stocker R (2002) Selective and sensitive measurement of vitamin C, ubiquinol-10 and other low-molecular-weight antioxidants. In Free Radicals - A Practical Approach, pp. 271-286 [NA Punchard and FJ Kelly, editors]. Oxford: Oxford University Press.

Newham DJ, Mills KR, Quigley BM \& Edwards RH (1983) Pain and fatigue after concentric and eccentric muscle contractions. Clin Sci (Lond) 64, 55-62.

Nieman DC, Henson DA, McAnulty SR, McAnulty L, Swick NS, Utter AC, Vinci DM, Opiela SJ \& Morrow JD (2002) Influence of vitamin $\mathrm{C}$ supplementation on oxidative and immune changes after an ultramarathon. J Appl Physiol 92, 1970-1977.

Niess AM, Dickhuth HH, Northoff H \& Fehrenbach E (1999) Free radicals and oxidative stress in exercise - immunological aspects. Exerc Immunol Rev 5, 22-56.

Pattwell D, Ashton T, McArdle A, Griffiths RD \& Jackson MJ (2003) Ischaemia and reperfusion of skeletal muscle leads to appearance of a stable lipid free radical in the circulation. Am J Physiol 284H, H2400-H2404.

Podmore ID, Griffiths HR, Herbert KE, Mistry N, Mistry P \& Lunec J (1998) Vitamin C exhibits pro-oxidant properties. Nature 392, 559.

Powers SK, DeRuisseau KC, Quindry J \& Hamilton KL (2004) Dietary antioxidants and exercise. J Sports Sci 22, 81-94.

Richard MJ, Guiraud P, Meo J \& Favier A (1992) High-performance liquid chromatographic separation of malondialdehyde-thiobarbituric acid adduct in biological materials (plasma and human cells) using a commercially available reagent. J Chromatogr 577, $9-18$.

Thompson D, Williams C, Kingsley M, Nicholas CW, Lakomy HK, McArdle F \& Jackson MJ (2001) Muscle soreness and damage parameters after prolonged intermittent shuttle-running following acute vitamin $\mathrm{C}$ supplementation. Int J Sports Med 22, $68-75$.

Thompson D, Williams C, McGregor SJ, Nicholas CW, McArdle F, Jackson MJ \& Powell JR (2001) Prolonged vitamin C supplementation and recovery from demanding exercise. Int J Sport Nutr Exerc Metab 11, 466-481. 